\title{
A Mathematical Model of Horizontal Averaged Groundwater Pollution Measurement with Several Substances due to Chemical Reaction
}

\author{
Jirapud Limthanakul ${ }^{1}$, Nopparat Pochai ${ }^{2, *}$ \\ ${ }^{1}$ Department of Mathematics, Faculty of Science, King Mongkut's Institute of Technology Ladkrabang, Bangkok 10520, Thailand \\ ${ }^{2}$ Centre of Excellence in Mathematics, Commission on Higher Education(CHE), Si Ayutthaya Road, Bangkok 10400, Thailand
}

Received June 11, 2020; Revised September 25, 2020; Accepted October 19, 2020

Cite This Paper in the following Citation Styles

(a): [1] Jirapud Limthanakul, Nopparat Pochai , "A Mathematical Model of Horizontal Averaged Groundwater Pollution Measurement with Several Substances due to Chemical Reaction," Mathematics and Statistics, Vol. 8, No. 6, pp. 645-655, 2020. DOI: 10.13189/ms.2020.080604.

(b): Jirapud Limthanakul, Nopparat Pochai, (2020). A Mathematical Model of Horizontal Averaged Groundwater Pollution Measurement with Several Substances due to Chemical Reaction. Mathematics and Statistics, 8(6), 645-655. DOI: 10.13189/ms.2020.080604.

Copyright $@ 2020$ by authors, all rights reserved. Authors agree that this article remains permanently open access under the terms of the Creative Commons Attribution License 4.0 International License

\begin{abstract}
Chloride is a well-known chemical compound that is very useful in industry and agricultural. Chloride can be transformed to hypochlorite, chlorite, chlorate and perchlorate, chloride and their substances are not dangerous if they are used in the optimal level. Groundwater containing contamination chloride and their substances impacts human health, for example, drinking water contaminated chloride exceed $250 \mathrm{mg} / \mathrm{L}$ can cause heart problems and lead to high blood pressure. To avoid this problem, we used mathematical models to explain groundwater contamination with chloride and their substances. Transient groundwater flow model provides the hydraulic head of groundwater. In this model we will get the level of groundwater, next, we need to find its velocity and direction by using the result in first model put into second model. Groundwater velocity model provides $x$ - and $z$-direction vector in groundwater, after computation we will plugin the result into the last model to approximate the chloride concentration in groundwater. Groundwater contamination dispersion model provides chloride, hypochlorite, chlorite, chlorate and perchlorate concentration. The proposed explicit finite difference techniques are used to approximate the model solution. Explicit method was used to solved hydraulic head model. Forward space described groundwater velocity model. Forward time and central space used to predict transient groundwater contaminated models. The simulations can be used to indicate when each simulated zone becomes a hazardous zone or a protection zone.
\end{abstract}

Keywords Finite Difference Method, Forward Space Technique, Forward Time Centred Space Two-dimensional

\section{Introduction}

Nowadays, we can say that water is an important part of life, whether in daily life, agricultural and industry. The water pollution problem is created by above activity, we can found the contaminated water from the natural source, contaminated water has so much effect, it can cause many life diseases and problems. Nitrates or nitrites in water contaminates drinking water can impacts human health by decreasing blood cell ability to carry oxygen, which can be linked to blue baby syndrome [2], this is one of the effects of contaminated water. In this research we talk about the effect of chloride and their substance.

Chloride occurs naturally in groundwater but is found in greater concentrations in seawater. It generally combines with sodium, calcium, or magnesium. For example, sodium chloride $(\mathrm{NaCl})$ is formed when chloride and sodium combine.

The other forms of chloride do not come from the only combination of other substances, the oxidation numbers or oxidation states is the well-know process to obtained a new form of chemical compound, for example, Chloride can be changed to hypochlorite $(\mathrm{ClO})$ if the oxidation number increase by 1 or chlorite if added by 3 .

\begin{tabular}{|c|c|c|c|c|c|}
\hline oxidation state & -1 & +1 & +3 & +5 & +7 \\
\hline anion name & chloride & hypochlorite & chlorite & chlorate & perchlorate \\
\hline formula & $\mathrm{Cl}^{-}$ & $\mathrm{ClO}^{-}$ & $\mathrm{ClO}_{2}^{-}$ & $\mathrm{ClO}_{3}^{-}$ & $\mathrm{ClO}_{4}^{-}$ \\
\hline
\end{tabular}

Figure 1. The other form of chloride in any state

They are substances used by the body to help it work well. Although chlorides are harmless at low levels, well water high in sodium chloride can damage plants if used for gardening or 
irrigation, and give drinking water an unpleasant taste. Sodium chloride is high corrosivity, which will also damage plumbing, appliances, and water heaters, causing toxic metals to leach into your water. It can complicate existing heart problems and contribute to high blood pressure when ingested in excess [1].

\begin{tabular}{|c|l|}
\hline chloride & can cause heart problems and contribute to high blood pressure \\
\hline hypochlorite & can cause bladder cancer \\
\hline chlorite & $\begin{array}{l}\text { can cause shortness of breath and other respiratory problems } \\
\text { because of damage to the substances in blood that carry oxygen } \\
\text { throughout the body }\end{array}$ \\
\hline chlorate & $\begin{array}{l}\text { chlorate is toxic, doses of a few grams of chlorate are lethal, there is a } \\
\text { direct toxicity to the proximal renal tubule }\end{array}$ \\
\hline perchlorate & $\begin{array}{l}\text { at high concentrations perchlorate can affect the thyroid gland by } \\
\text { inhibiting the uptake of iodine }\end{array}$ \\
\hline
\end{tabular}

Figure 2. Chloride compound damage to health

There are many dangers of chloride, but we can prevent amounts of them, from exceeding standards by management based on mathematical models. Yamashita and Sugio (2000) developed a model of advection dispersion and biochemical reactions [3]. Pochai and Kraychang (2016) solved a two mathematical models for simulating water pollutant level and pollution control in a connected reservoir system [7]. Gardenas (2005) talks about a two-dimensional modeling approach to improve fertigation strategies and soil types on nitrate leaching potential [4]. The one-dimensional advection-diffusion equation with constant coefficients have been solved by Dehghan (2004) [5]. Kewalee and Pochai (2018) described the governing equation in the air quality model in three-dimensional advection-diffusion equations with time dependence [6].

In this paper, we measured groundwater that has been contaminated by chloride on a landfill. The simulation required data concerned with the groundwater flow velocity of the current points and any time in the domain. The groundwater flow velocity can be obtained by using transient twodimensional groundwater flow model. We used the transient two-dimensional advection diffusion equation to approximated the chloride concentration. The finite elements [19, 20, 21] and finite difference methods $[16,17,18]$ are the most popular numerical solution techniques. Among of these, finite difference methods, including both explicit and implicit schemes, are mostly used for two-dimensional domain such as in latitude and longitude stream.

\section{Chloride pollutant measure models}

\subsection{Transient groundwater flow model}

The governing equation of a latitudinally integrated Darcy's flow in a two-dimensional advection-diffusion equation [8],

$S \frac{\partial H(x, z, t)}{\partial t}=\frac{\partial}{\partial x}\left(K_{x} \frac{\partial H(x, z, t)}{\partial x}\right)+\frac{\partial}{\partial z}\left(K_{z} \frac{\partial H(x, z, t)}{\partial z}\right)$

where $H(x, z, t)$ is the hydraulic head(metre), $S$ matrix of specific storage(1/metre), $L_{x}$ is the considered area length, $L_{z}$ is the depth of considered groundwater area, $T$ is the stationary time of simulation as shown in Figure 1. The hydraulic conductivity(metre/day) component in the $x, z$ directions are denoted by $K_{x}, K_{z}$, respectively. Assuming that the soil topography in the considered area is homogeneous, these mean that the hydraulic conductivity are constant.

$$
S \frac{\partial H(x, z, t)}{\partial t}=K_{x} \frac{\partial^{2} H(x, z, t)}{\partial x}+K_{z} \frac{\partial^{2} H(x, z, t)}{\partial z} .
$$

for all $(x, z, t) \in \Omega$ such that $\Omega=\left[0, L_{x}\right] \times\left[0, L_{z}\right] \times[0, T]$.

\subsection{Initial and boundary condition of transient groundwater flow model}

\subsubsection{The initial condition of transient groundwater flow model}

The initial condition is defined by an interpolation function of measured raw data as be low

$$
H(x, z, 0)=h(x, z),
$$

where $h(x, z)$ is a given potential hydraulic head function.

\subsubsection{The boundary conditions}

The top, right and bottom boundary conditions are assumed by the average rate of change of hydraulic head around the top, right and bottom boundaries. The left boundary condition is assumed by the interpolation function of measured raw data in the considered landfill as shown in Figure 1. The boundary condition, are also assumed by

$$
\begin{gathered}
H(x, z, t)=f_{L}(z), \text { for all } z \in\left[0, I_{z}\right] \text { and } x=0, \\
\frac{\partial H(x, z, t)}{\partial z}=f_{T}(x), \text { for all } x \in\left[0, I_{x}\right] \text { and } z=M_{z}, \\
\frac{\partial H(x, z, t)}{\partial x}=f_{R}(z), \text { for all } z \in\left[0, I_{z}\right] \text { and } x=M_{x}, \\
\frac{\partial H(x, z, t)}{\partial z}=f_{B}(x), \text { for all } x \in\left[0, I_{x}\right] \text { and } z=0,
\end{gathered}
$$

where $f_{L}(z), f_{T}(x), f_{R}(z)$ and $f_{B}(x)$ are the boundary source of hydraulic head on the left boundary domain. The rate of change hydraulic head with respect to domain boundaries around the top, the bottom and the right bottom around the considered area are shown in Figure 2.

\subsection{Groundwater flow velocity model}

We can obtain that the groundwater flow velocity in $x$ direction is a decreasing rate of change of the hydraulic head $x$-direction,

$$
u=-\frac{\partial H}{\partial x}
$$

Similarly, the groundwater flow velocity in $z$-direction is a decreasing rate of change of the hydraulic head in $z$-direction,

$$
w=-\frac{\partial H}{\partial z}
$$




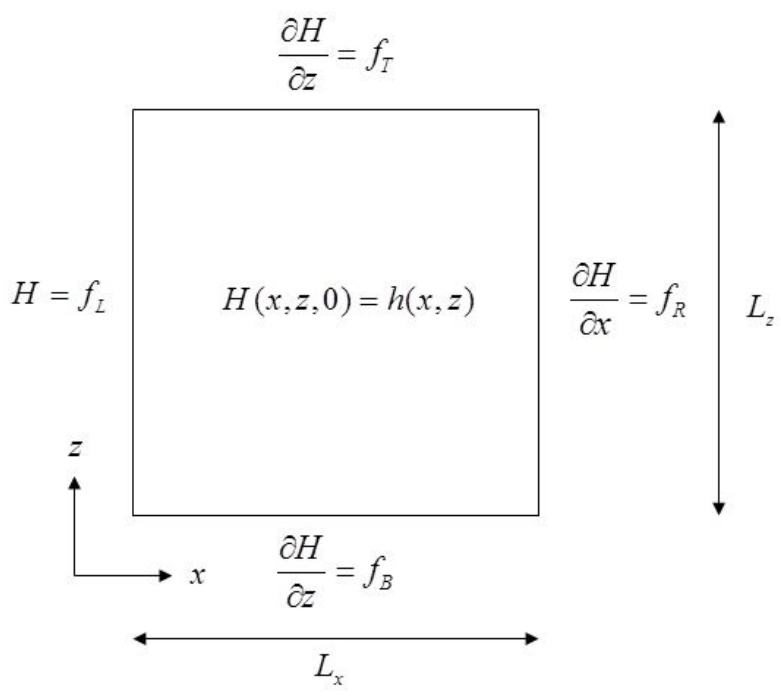

Figure 3. General structure of problem domain.

\subsection{Chloride Compound Dispersion Models}

\subsubsection{A total chloride dispersion model}

The pollutant concentration measurement of total chloride in surface water can be describe by a two-dimensional advectiondiffusion reaction equation.

$$
\begin{array}{r}
\frac{\partial c(x, z, t)}{\partial t}+u \frac{\partial c(x, z, t)}{\partial x}+w \frac{\partial c(x, z, t)}{\partial z} \\
=D_{x} \frac{\partial^{2} c(x, z, t)}{\partial x^{2}}+D_{z} \frac{\partial^{2} c(x, z, t)}{\partial z^{2}}+Q-R c,
\end{array}
$$

for all $(x, z, t) \in \Omega$ such that $\Omega=\left[0, L_{x}\right] \times\left[0, L_{z}\right] \times[0, T]$, where $c(x, z, t)$ is total chloride pollutant concentration of groundwater $\left(\mathrm{kg} / \mathrm{m}^{3}\right), D_{x}$ and $D_{z}$ are the diffusion coefficient in $x$ - and $z$-directions, $u(x, z, t), w(x, z, t)$ are the groundwater flow velocity in the $x$ - and $z$-directions, and $R$ is transformed chloride rate.

\subsection{Initial condition of the total chloride disper- sion model}

The chloride dispersion is described with conditions in the following sections, where the potential groundwater pollutant concentration in the consider area is described by

$$
c(x, z, 0)=c_{0}(x, z) . \text { for all }(x, z) \in \Omega
$$

where $c_{0}(x, z)$ is a averaged potential total chloride concentration in the considered area.

\subsection{Boundary condition of total chloride disper- sion model}

The left boundary condition is assumed by the interpolation function of measured raw data at the considered landfill. The top, right and bottom boundary conditions are assumed by the averaged rates of change of pollutant concentration around the top, right and bottom boundaries. The boundary conditions are also assumed by

$$
\begin{aligned}
& c\left(L_{z}, x, t\right)=g_{N}(x), \text { for all } x \in\left[k_{1} L_{x}, k_{2} L_{x}\right], \\
& \frac{\partial c(z, 0, t)}{d x}=g_{L}(z), \quad \text { for all } z \in\left[0, L_{z}\right] \text { and } t \in[0, T],
\end{aligned}
$$

$$
\begin{aligned}
\frac{\partial c\left(L_{z}, x, t\right)}{d z}=g_{T}(x), & \text { for all } x \in\left[0, k_{1} L_{x}\right) \cup\left(k_{2} L_{x}, L_{x}\right] \\
& \text { and } t \in[0, T], \\
\frac{\partial c\left(z, L_{x}, t\right)}{d x}=g_{R}(z), & \text { for all } z \in\left[0, L_{x}\right] \text { and } t \in[0, T] \\
\frac{\partial c(x, 0, t)}{d z}=g_{B}(x), & \text { for all } x \in\left[0, L_{x}\right] \text { and } t \in[0, T]
\end{aligned}
$$

where $k_{1} L_{x}, k_{2} L_{x}$ are referred to as the range area of the total chloride pollutant area source, and $g_{L}(z), g_{T}(x), g_{R}(z)$ and $g_{B}(x)$ are the rate of change of the total chloride concentration with respect to distance around the top, the bottom and the right boundaries along the considered area, respectively.

\subsubsection{A hypochlorite dispersion model}

The total chloride is transformed to be the hypochlorite. The hypochlorite dispersion model is described by

$$
\frac{\partial \phi}{\partial t}+u \frac{\partial \phi}{\partial x}+v \frac{\partial \phi}{\partial z}=D 1_{x} \frac{\partial^{2} \phi}{\partial x^{2}}+D 1_{z} \frac{\partial^{2} \phi}{\partial z^{2}}+Q+R_{1} R c
$$

for all $(x, z, t) \in \Omega$ such that $\Omega=\left[0, L_{x}\right] \times\left[0, L_{z}\right] \times[0, T]$, where $\phi(x, z, t)$ is hypochlorite pollutant concentration of groundwater $\left(\mathrm{kg} / \mathrm{m}^{3}\right), D 1_{x}, D 1_{z}$ are the diffusion coefficient in $x$ - and $z$-directions, $u(x, z, t), w(x, z, t)$ are the groundwater flow velocity in the $x$ - and $z$-directions and $R_{1}$ is transformed hypochlorite rate.

\subsection{Initial condition of hypochlorite dispersion model}

Dispersion of hypochlorite with the following conditions, if the potential hypochlorite concentration in the consider area is described by

$$
\phi(x, z, 0)=f_{\phi}(x, z) . \text { for all }(x, z) \in \Omega
$$

where $f_{\phi}(x, z)$ is a averaged potential hypochlorite concentration in the considered area. 


\subsection{Boundary condition of hypochlorite disper- sion model}

The rate of change of the pollutant concentration along the domain boundaries are assumed to be:

$$
\begin{gathered}
\frac{\partial \phi(0, z, t)}{\partial x}=g 1_{L}(z), \text { for all } z \in\left[0, L_{z}\right] \text { and } t \in[0, T] \\
\frac{\partial \phi\left(x, L_{z}, t\right)}{\partial z}=g 1_{T}(x), \text { for all } x \in\left[0, L_{x}\right] \text { and } t \in[0, T], \\
\frac{\partial \phi\left(L_{x}, z, t\right)}{\partial x}=g 1_{R}(z), \text { for all } z \in\left[0, L_{x}\right] \text { and } t \in[0, T] \\
\frac{\partial \phi(x, 0, t)}{\partial z}=g 1_{B}(x), \text { for all } x \in\left[0, L_{x}\right] \text { and } t \in[0, T]
\end{gathered}
$$

where $g 1_{L}(z), g 1_{T}(x), g 1_{R}(z)$ and $g 1_{B}(x)$ are the rate of change of the hypochlorite concentration with respect distance around the top, the bottom and the right boundaries along the considered area, respectively.

\subsubsection{A chlorite dispersion model}

The model of chlorite dispersion model can be described by $\frac{\partial \eta}{\partial t}+u \frac{\partial \eta}{\partial x}+v \frac{\partial \eta}{\partial z}=D 2_{x} \frac{\partial^{2} \eta}{\partial x^{2}}+D 2_{z} \frac{\partial^{2} \eta}{\partial z^{2}}+Q+R_{2} R c$

for all $(x, z, t) \in \Omega$ such that $\Omega=\left[0, L_{x}\right] \times\left[0, L_{z}\right] \times$ $[0, T]$, where $\eta(x, z, t)$ is chlorite pollutant concentration of groundwater $\left(\mathrm{kg} / \mathrm{m}^{3}\right), D 2_{x}, D 2_{z}$ are the diffusion coefficient in $x$ - and $z$-directions, $u(x, z, t), w(x, z, t)$ are the groundwater flow velocity in the $x$ - and $z$-directions, $R_{2}$ is transformed chlorite rate.

\subsection{Initial condition of chlorite dispersion model}

Dispersion of chlorite with the following conditions, if the potential chlorite concentration in the consider area is described by

$$
\eta(x, z, 0)=f_{\eta}(x, z) . \text { for all }(x, z) \in \Omega
$$

where $f_{\eta}(x, z)$ is a averaged potential chlorite concentration in the considered area.

\subsection{Boundary condition of chlorite dispersion model}

Similarly, the rate of change are assumed by $g 2_{L}(z)$, $g 2_{T}(x), g 2_{R}(z)$ and $g 2_{B}(x)$.

\subsubsection{A chlorate dispersion model}

The model of chlorate dispersion model can be described by $\frac{\partial \sigma}{\partial t}+u \frac{\partial \sigma}{\partial x}+v \frac{\partial \sigma}{\partial z}=D 3_{x} \frac{\partial^{2} \sigma}{\partial x^{2}}+D 3_{z} \frac{\partial^{2} \sigma}{\partial z^{2}}+Q+R_{3} R c$ for all $(x, z, t) \in \Omega$ such that $\Omega=\left[0, L_{x}\right] \times\left[0, L_{z}\right] \times$ $[0, T]$, where $\sigma(x, z, t)$ is chlorate pollutant concentration of groundwater $\left(\mathrm{kg} / \mathrm{m}^{3}\right), D 3_{x}, D 3_{z}$ are the diffusion coefficient in $x$ - and $z$-directions, $u(x, z, t), w(x, z, t)$ are the groundwater flow velocity in the $x$ - and $z$-directions, $R_{3}$ is transformed chlorate rate.

\subsection{Initial condition of chlorate dispersion model}

Dispersion of chlorate with following conditions, if the potential chlorate concentration in the consider area is described by

$$
\sigma(x, z, 0)=f_{\sigma}(x, z) \text {. for all }(x, z) \in \Omega
$$

where $f_{\sigma}(x, z)$ is a averaged potential chlorate concentration in the considered area.

\subsection{Boundary condition of chlorate dispersion model}

Similarly, the rate of change are assumed by $g 3_{L}(z)$, $g 3_{T}(x), g 3_{R}(z)$ and $g 3_{B}(x)$.

\subsubsection{A perchlorate dispersion model}

The model of perchlorate dispersion model can be described by

$$
\frac{\partial \xi}{\partial t}+u \frac{\partial \xi}{\partial x}+v \frac{\partial \xi}{\partial z}=D 4_{x} \frac{\partial^{2} \xi}{\partial x^{2}}+D 4_{z} \frac{\partial^{2} \xi}{\partial z^{2}}+Q+R_{4} R c
$$

for all $(x, z, t) \in \Omega$ such that $\Omega=\left[0, L_{x}\right] \times\left[0, L_{z}\right] \times$ $[0, T]$, where $\xi(x, z, t)$ is perchlorate pollutant concentration of groundwater $\left(\mathrm{kg} / \mathrm{m}^{3}\right), D 4_{x}, D 4_{z}$ are the diffusion coefficient in $x$ - and $z$-directions, $u(x, z, t), w(x, z, t)$ are the groundwater flow velocity in the $x$ - and $z$-directions, $R_{4}$ is transformed perchlorate rate.

\subsection{Initial condition of perchlorate dispersion model}

Dispersion of perchlorate with following conditions, if the potential perchlorate concentration in the consider area is described by

$$
\xi(x, z, 0)=f_{\xi}(x, z) \text {. for all }(x, z) \in \Omega
$$

where $f_{\xi}(x, z)$ is a averaged potential perchlorate concentration in the considered area.

\subsection{Boundary condition of perchlorate disper- sion model}

Similarly, the rate of change are assumed by $g 4_{L}(z)$, $g 4_{T}(x), g 4_{R}(z)$ and $g 4_{B}(x)$. 


\section{Numerical techniques}

In this paper, we will propose finite difference methods to the transient groundwater model by using the forward time central space method. We now discretize the domain by dividing the interval $\left[0, L_{x}\right]$ and $\left[0, L_{z}\right]$ into $M_{x}$ and $M_{z}$ subintervals such that $M_{x} \Delta x=L_{x}, M_{z} \Delta z=L_{z}$ and the time interval $[0, T]$ into $N$ subintervals such that $N \Delta t=T$. The grid points $\left(x_{j}, z_{i}, t_{n}\right)$ are defined by $x_{j}=j \Delta x$ for all $j=1,2, \ldots, M_{x}$, $z_{i}=i \Delta z$ for all $i=1,2, \ldots, M_{z}$ and $t_{n}=n \Delta t$ for all $n=1,2, \ldots, T$. We can then approximate $H\left(x_{j}, z_{i}, t_{n}\right)$ by $H_{i, j}^{n}$, value of the difference approximation of $H(x, z, t)$ at point $x=j \Delta x, z=i \Delta z$ and $t=n \Delta t$, where $0 \leq j \leq$ $M_{x}, 0 \leq i \leq M_{z}$ and $0 \leq n \leq N$ which $M_{x}, M_{z}$ and $N$ are positive integers.

\subsection{Explicit finite difference method for two- dimensional groundwater flow model}

Taking the central difference in space and forward difference in time into scheme each terms of Eq. (2.2), we have

$$
\begin{aligned}
H(x, z, t) & \simeq H_{i, j}^{n}, \\
\left.\frac{\partial H}{\partial t}\right|_{\left(x_{i}, z_{j}, t_{n}\right)} & \simeq \frac{H_{i, j}^{n+1}-H_{i, j}^{n}}{\Delta t} \\
\left.\frac{\partial^{2} H}{\partial x^{2}}\right|_{\left(x_{i}, z_{j}, t_{n}\right)} & \simeq \frac{H_{i, j-1}^{n}-2 H_{i, j}^{n}+H_{i, j+1}^{n}}{(\Delta x)^{2}} \\
\left.\frac{\partial^{2} H}{\partial z^{2}}\right|_{\left(x_{i}, z_{j}, t_{n}\right)} & \simeq \frac{H_{i-1, j}^{n}-2 H_{i, j}^{n}+H_{i+1, j}^{n}}{(\Delta z)^{2}} .
\end{aligned}
$$

Substituting Eqs.(3.1)-(3.4) into Eq.(2.2), we get the finite equation,

$$
\begin{aligned}
S\left(\frac{H_{i, j}^{n+1}-H_{i, j}^{n}}{\Delta t}\right) & =K_{x}\left(\frac{H_{i, j-1}^{n}-2 H_{i, j}^{n}+H_{i, j+1}^{n}}{(\Delta x)^{2}}\right) \\
& +K_{z}\left(\frac{H_{i-1, j}^{n}-2 H_{i, j}^{n}+H_{i+1, j}^{n}}{(\Delta z)^{2}}\right),
\end{aligned}
$$

for all $i=1,2,3, \ldots, M_{x}, j=1,2,3, \ldots, M_{z}$ and $n=$ $1,2,3, \ldots, N-1$. Then the explicit finite difference equation becomes

$$
\begin{aligned}
H_{i, j}^{n+1} & =\alpha H_{i, j-1}^{n}+\alpha H_{i, j+1}^{n}+(1-2 \alpha-2 \beta) H_{i, j}^{n} \\
& +\beta H_{i-1, j}^{n}+\beta H_{i+1, j}^{n}
\end{aligned}
$$

where $\alpha=\frac{K_{x}(\Delta t)}{S(\Delta x)^{2}}$ and $\beta=\frac{K_{z}(\Delta t)}{S(\Delta z)^{2}}$, for all $i=$ $1,2, \ldots, M_{x}-1$ and $j=1,2, \ldots, M_{z}-1$.

The forward space technique is used to approximate fictitious points on the boundaries solution such as,

$$
\begin{aligned}
H_{i, M_{x}+1}^{n} & =H_{i, M_{x}}^{n}+f_{R}(z) \Delta x \\
H_{-1, j}^{n} & =H_{0, j}^{n}-f_{B}(x) \Delta z \\
H_{M_{z}, j}^{n+1} & =H_{M_{z}, j}^{n}+f_{T}(x) \Delta z .
\end{aligned}
$$

\subsection{Finite difference method for groundwater flow velocity model}

Taking the forward difference in space into Eq.(2.8) and Eq.(2.9), we have

$$
\begin{aligned}
v(x, z, t) & \simeq v_{i, j}^{n}, \\
w(x, z, t) & \simeq w_{i, j}^{n}, \\
\left.\frac{\partial H}{\partial x}\right|_{\left(x_{i}, z_{j}, t_{n}\right)} & \simeq \frac{H_{i, j+1}^{n}-H_{i, j}^{n}}{\Delta x}, \\
\left.\frac{\partial H}{\partial z}\right|_{\left(x_{i}, z_{j}, t_{n}\right)} & \simeq \frac{H_{i+1, j}^{n}-H_{i, j}^{n}}{\Delta z} .
\end{aligned}
$$

Substituting Eqs.(3.12) - (3.13) into (2.8) and (2.9), we get the finite equation,

$$
v_{i, j}^{n}=-\frac{1}{\Delta x}\left(H_{i, j+1}^{n}-H_{i, j}^{n}\right)
$$

and

$$
w_{i, j}^{n}=-\frac{1}{\Delta z}\left(H_{i+1, j}^{n}-H_{i, j}^{n}\right)
$$

\subsection{Explicit finite difference method for two- dimensional groundwater pollution disper- sion model}

In this section, the considered domain is defined in a similar grid spacing as the previous. We will employ forward time central space difference scheme (FTCS) to Eqs.(2.10)

$$
\begin{aligned}
c(x, z, t) & \simeq C_{i, j}^{n}, \\
\left.\frac{\partial c}{\partial t}\right|_{\left(x_{i}, z_{j}, t_{n}\right)} & \simeq \frac{C_{i, j}^{n+1}-C_{i, j}^{n}}{\Delta t}, \\
\left.\frac{\partial c}{\partial x}\right|_{\left(x_{i}, z_{j}, t_{n}\right)} & \simeq \frac{C_{i, j-1}^{n}-C_{i, j+1}^{n}}{2 \Delta x}, \\
\left.\frac{\partial c}{\partial z}\right|_{\left(x_{i}, z_{j}, t_{n}\right)} & \simeq \frac{C_{i-1, j}^{n}-C_{i+1, j}^{n}}{2 \Delta z}, \\
\left.\frac{\partial^{2} c}{\partial x^{2}}\right|_{\left(x_{i}, z_{j}, t_{n}\right)} & \simeq \frac{C_{i, j-1}^{n}-2 C_{i, j}^{n}+C_{i, j+1}^{n}}{(\Delta x)^{2}}, \\
\left.\frac{\partial^{2} c}{\partial z^{2}}\right|_{\left(x_{i}, z_{j}, t_{n}\right)} & \simeq \frac{C_{i-1, j}^{n}-2 C_{i, j}^{n}+C_{i+1, j}^{n}}{(\Delta z)^{2}} .
\end{aligned}
$$

Substituting Eqs.(3.16) - (3.21) in chloride dispersion models,

\subsection{An explicit the forward time centered space method for a total chloride model}

$$
\begin{aligned}
C_{i, j}^{n+1} & =\left(\tau_{1}+\tau_{2}\right) C_{i-1, j}^{n}+\left(1-2 \lambda_{1}-2 \tau_{1}\right) C_{i, j}^{n} \\
& +\left(\tau_{1}-\tau_{2}\right) C_{i+1, j}^{n}+\left(\lambda_{1}-\lambda_{2}\right) C_{i, j+1}^{n} \\
& +\left(\lambda_{1}+\lambda_{2}\right) C_{i, j-1}^{n}+Q \Delta t-\Delta t R C_{i, j}^{n},
\end{aligned}
$$

for all $i=1,2,3, \ldots, M_{x}, j=1,2,3, \ldots, M_{z}$ and $n=$ $1,2,3, \ldots, N-1$. 
where $\lambda_{1}=\frac{D_{x} \Delta t}{(\Delta x)^{2}}, \lambda_{2}=\frac{u_{i, j}^{n} \Delta t}{2 \Delta x}, \tau_{1}=\frac{D_{z} \Delta t}{(\Delta z)^{2}}$. and $\tau_{2}=$ $\frac{w_{i, j}^{n} \Delta t}{2 \Delta z}$.

The forward space technique is used to approximate fictitious points on the boundaries solution such as,

$$
\begin{aligned}
C_{i,-1}^{n} & =C_{i, 0}^{n}-g_{W}(z) \Delta x, \\
C_{i, M_{x}+1}^{n} & =C_{i, M_{x}}^{n}+g_{R}(z) \Delta x, \\
C_{-1, j}^{n} & =C_{0, j}^{n}-g_{B}(x) \Delta z, \\
C_{M_{z}+1, j}^{n} & =C_{M_{z}, j}^{n}+g_{T}(x) \Delta z .
\end{aligned}
$$

\subsection{An explicit the forward time centered space method for a hypochlorite}

Taking the central difference in space and forward difference in time into scheme each terms of Eq. (2.18), we have

$$
\begin{aligned}
\phi_{i, j}^{n+1} & =\left(\tau_{3}+\tau_{4}\right) \phi_{i-1, j}^{n}+\left(1-2 \lambda_{3}-2 \tau_{3}\right) \phi_{i, j}^{n} \\
& +\left(\tau_{3}-\tau_{4}\right) \phi_{i+1, j}^{n}+\left(\lambda_{3}-\lambda_{4}\right) \phi_{i, j+1}^{n} \\
& +\left(\lambda_{3}+\lambda_{4}\right) \phi_{i, j-1}^{n}+Q \Delta t+\Delta t R_{1} R C_{i, j}^{n},
\end{aligned}
$$

for all $i=1,2,3, \ldots, M_{x}, j=1,2,3, \ldots, M_{z}$ and $n=$ $1,2,3, \ldots, N-1$.

where $\lambda_{3}=\frac{D 1_{x} \Delta t}{(\Delta x)^{2}}, \lambda_{4}=\frac{u_{i, j}^{n} \Delta t}{2 \Delta x}, \tau_{3}=\frac{D 1_{z} \Delta t}{(\Delta z)^{2}}$. and $\tau_{4}=\frac{w_{i, j}^{n} \Delta t}{2 \Delta z}$.

The forward space technique is used to approximate fictitious points on the boundaries solution such as,

$$
\begin{aligned}
\phi_{i,-1}^{n} & =\phi_{i, 0}^{n}-g 1_{W}(z) \Delta x, \\
\phi_{i, M_{x}+1}^{n} & =\phi_{i, M_{x}}^{n}+g 1_{R}(z) \Delta x, \\
\phi_{-1, j}^{n} & =\phi_{0, j}^{n}-g 1_{B}(x) \Delta z, \\
\phi_{M_{z}+1, j}^{n} & =\phi_{M_{z}, j}^{n}+g 1_{T}(x) \Delta z .
\end{aligned}
$$

\subsection{An explicit the forward time centered space method for a chlorite}

Taking the central difference in space and forward difference in time into scheme each terms of Eq. (2.23), we have

$$
\begin{aligned}
\eta_{i, j}^{n+1} & =\left(\tau_{5}+\tau_{6}\right) \eta_{i-1, j}^{n}+\left(1-2 \lambda_{5}-2 \tau_{5}\right) \eta_{i, j}^{n} \\
& +\left(\tau_{5}-\tau_{6}\right) \eta_{i+1, j}^{n}+\left(\lambda_{5}-\lambda_{6}\right) \eta_{i, j+1}^{n} \\
& +\left(\lambda_{5}+\lambda_{6}\right) \eta_{i, j-1}^{n}+Q \Delta t+\Delta t R_{2} R C_{i, j}^{n},
\end{aligned}
$$

for all $i=1,2,3, \ldots, M_{x}, j=1,2,3, \ldots, M_{z}$ and $n=$ $1,2,3, \ldots, N-1$.

where $\lambda_{5}=\frac{D 2_{x} \Delta t}{(\Delta x)^{2}}, \lambda_{6}=\frac{u_{i, j}^{n} \Delta t}{2 \Delta x}, \tau_{5}=\frac{D 2_{z} \Delta t}{(\Delta z)^{2}}$. and $\tau_{6}=\frac{w_{i, j}^{n} \Delta t}{2 \Delta z}$.
The forward space technique is used to approximate fictitious points on the boundaries solution such as,

$$
\begin{aligned}
\eta_{i,-1}^{n} & =\eta_{i, 0}^{n}-g 2_{W}(z) \Delta x, \\
\eta_{i, M_{x}+1}^{n} & =\eta_{i, M_{x}}^{n}+g 2_{R}(z) \Delta x, \\
\eta_{-1, j}^{n} & =\eta_{0, j}^{n}-g 2_{B}(x) \Delta z, \\
\eta_{M_{z}+1, j}^{n} & =\eta_{M_{z}, j}^{n}+g 2_{T}(x) \Delta z .
\end{aligned}
$$

\subsection{An explicit the forward time centered space method for a chlorate}

Taking the central difference in space and forward difference in time into scheme each terms of Eq. (2.25), we have

$$
\begin{aligned}
\sigma_{i, j}^{n+1} & =\left(\tau_{7}+\tau_{8}\right) \sigma_{i-1, j}^{n}+\left(1-2 \lambda_{7}-2 \tau_{7}\right) \sigma_{i, j}^{n} \\
& +\left(\tau_{7}-\tau_{8}\right) \sigma_{i+1, j}^{n}+\left(\lambda_{7}-\lambda_{8}\right) \sigma_{i, j+1}^{n} \\
& +\left(\lambda_{7}+\lambda_{8}\right) \sigma_{i, j-1}^{n}+Q \Delta t+\Delta t R_{3} R C_{i, j}^{n},
\end{aligned}
$$

for all $i=1,2,3, \ldots, M_{x}, j=1,2,3, \ldots, M_{z}$ and $n=$ $1,2,3, \ldots, N-1$.

where $\lambda_{7}=\frac{D 3_{x} \Delta t}{(\Delta x)^{2}}, \lambda_{8}=\frac{u_{i, j}^{n} \Delta t}{2 \Delta x}, \tau_{7}=\frac{D 3_{z} \Delta t}{(\Delta z)^{2}}$. and $\tau_{8}=\frac{w_{i, j}^{n} \Delta t}{2 \Delta z}$.

The forward space technique is used to approximate fictitious points on the boundaries solution such as,

$$
\begin{aligned}
\sigma_{i,-1}^{n} & =\sigma_{i, 0}^{n}-g 3_{W}(z) \Delta x, \\
\sigma_{i, M_{x}+1}^{n} & =\sigma_{i, M_{x}}^{n}+g 3_{R}(z) \Delta x, \\
\sigma_{-1, j}^{n} & =\sigma_{0, j}^{n}-g 3_{B}(x) \Delta z, \\
\sigma_{M_{z}+1, j}^{n} & =\sigma_{M_{z}, j}^{n}+g 3_{T}(x) \Delta z .
\end{aligned}
$$

\subsection{An explicit the forward time centered space method for a perchlorate}

Taking the central difference in space and forward difference in time into scheme each terms of Eq. (2.27), we have

$$
\begin{aligned}
\xi_{i, j}^{n+1} & =\left(\tau_{9}+\tau_{10}\right) \xi_{i-1, j}^{n}+\left(1-2 \lambda_{9}-2 \tau_{9}\right) \xi_{i, j}^{n} \\
& +\left(\tau_{9}-\tau_{10}\right) \xi_{i+1, j}^{n}+\left(\lambda_{9}-\lambda_{10}\right) \xi_{i, j+1}^{n} \\
& +\left(\lambda_{9}+\lambda_{10}\right) \xi_{i, j-1}^{n}+Q \Delta t+\Delta t R_{4} R C_{i, j}^{n},
\end{aligned}
$$

for all $i=1,2,3, \ldots, M_{x}, j=1,2,3, \ldots, M_{z}$ and $n=$ $1,2,3, \ldots, N-1$.

where $\lambda_{9}=\frac{D 4_{x} \Delta t}{(\Delta x)^{2}}, \lambda_{10}=\frac{u_{i, j}^{n} \Delta t}{2 \Delta x}, \tau_{9}=\frac{D 4_{z} \Delta t}{(\Delta z)^{2}}$. and $\tau_{10}=\frac{w_{i, j}^{n} \Delta t}{2 \Delta z}$.

The forward space technique is used to approximate fictitious points on the boundaries solution such as,

$$
\begin{aligned}
\xi_{i,-1}^{n} & =\xi_{i, 0}^{n}-g 4_{W}(z) \Delta x, \\
\xi_{i, M_{x}+1}^{n} & =\xi_{i, M_{x}}^{n}+g 4_{R}(z) \Delta x, \\
\xi_{-1, j}^{n} & =\xi_{0, j}^{n}-g 4_{B}(x) \Delta z, \\
\xi_{M_{z}+1, j}^{n} & =\xi_{M_{z}, j}^{n}+g 4_{T}(x) \Delta z .
\end{aligned}
$$




\section{Numerical simulations}

A two-dimensional hydraulic head model provides hydraulic head. The computed hydraulic head is transformed to be the groundwater flow velocity by using the second model. The results from the second model will be input into the chloride dispersion models that provide chloride, hypochlorite, chlorite, chlorate and perchlorate concentration

\subsection{Numerical simulation of transient ground- water flow velocity}

A chloride compound dispersion model that provide the concentration of their substance, this computation need to using the groundwater flow velocity from the first model.

The two-dimensional of groundwater flow model, considering with area domain $1.0 \times 0.5 \mathrm{~km}$. Assume that the specific storage is $1 \mathrm{~m}^{-1}$ and the hydraulic conductivity in $x$ - and $z$ direction are 15 (m/day) and don't have source term. The grid spacing is $\Delta x=\Delta z=10 \mathrm{~m}$, time step $\Delta t=1$ day at time $T=3650$ day. The initial $h=0(\mathrm{~m})$ and boundary conditions $h_{T}=h_{R}=h_{B}=0$ and $h_{L}=0.06 z+10$. Consider the hydraulic head of groundwater by using the explicit method, we get the approximated groundwater pollutant concentration as shown in Table 1 and Figures 3-4.

Table 1. The approximated hydraulic head(m) where $z=50 \mathrm{~m}$.

\begin{tabular}{lllllll}
\hline \multicolumn{7}{c}{$H(x, z, t)$} \\
\hline$t$ & $x=0$ & $x=20$ & $x=40$ & $x=60$ & $x=80$ & $x=90$ \\
\hline 3 & 36.4000 & 33.0498 & 29.7399 & 26.5099 & 23.3977 & 21.8976 \\
5 & 36.4000 & 33.7674 & 31.1538 & 28.5782 & 26.0593 & 24.8267 \\
7 & 36.4000 & 34.1465 & 31.9047 & 29.6864 & 27.5034 & 26.4286 \\
10 & 36.4000 & 34.4811 & 32.5693 & 30.6719 & 28.7960 & 27.8684 \\
\hline
\end{tabular}

Next, input the hydraulic head into the second model to approximate groundwater velocity in (2.8) and (2.9) by using central space. Then, we get the approximated groundwater flow velocity as shown in Tables 2-3 and Figure 5.

Table 2. The approximated groundwater flow velocity in $x$-direction(m/day) where $z=50 \mathrm{~m}$.

\begin{tabular}{lllllll}
\hline \multicolumn{7}{c}{$u(x, z, t)$} \\
\hline$t$ & $x=0$ & $x=20$ & $x=40$ & $x=60$ & $x=80$ & $x=90$ \\
\hline 3 & 0.1490 & 0.1470 & 0.1411 & 0.1338 & 0.1260 & 0.1220 \\
5 & 0.1212 & 0.1194 & 0.1143 & 0.1082 & 0.1020 & 0.0989 \\
7 & 0.1096 & 0.1080 & 0.1030 & 0.0972 & 0.0913 & 0.0885 \\
10 & 0.1029 & 0.1013 & 0.0964 & 0.0907 & 0.0850 & 0.0823 \\
\hline
\end{tabular}

Table 3. The approximated groundwater flow velocity in $z$-direction(m/day) where $z=50 \mathrm{~m}$.

\begin{tabular}{lllllll}
\hline \multicolumn{7}{c}{$w(x, z, t)$} \\
\hline$t$ & $x=0$ & $x=20$ & $x=40$ & $x=60$ & $x=80$ & $x=90$ \\
\hline 3 & -0.0600 & -0.0472 & -0.0366 & -0.0287 & -0.0228 & -0.0205 \\
5 & -0.0600 & -0.0473 & -0.0369 & -0.0291 & -0.0233 & -0.0211 \\
7 & -0.0600 & -0.0473 & -0.0369 & -0.0291 & -0.0234 & -0.0212 \\
10 & -0.0600 & -0.0473 & -0.0369 & -0.0291 & -0.0234 & -0.0212 \\
\hline
\end{tabular}

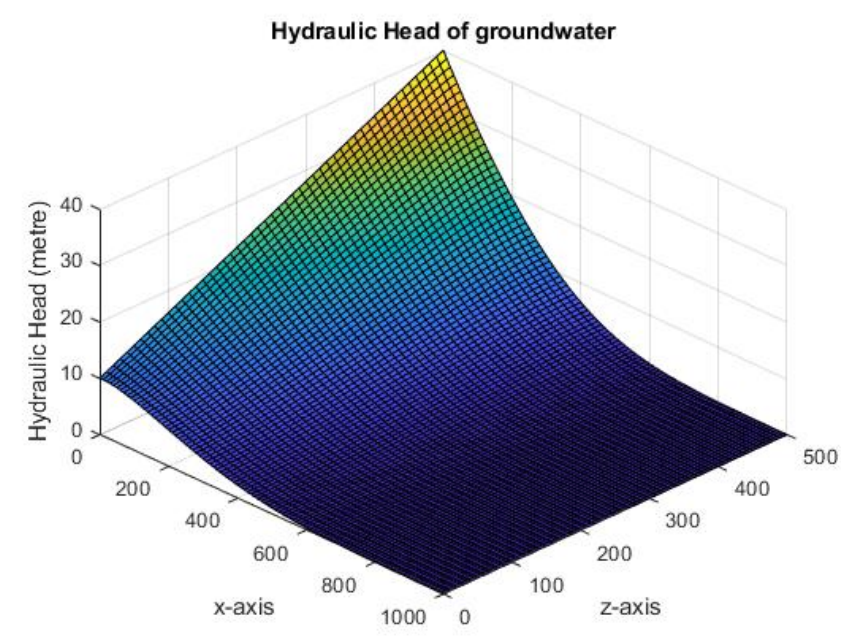

Figure 4. Hydraulic head of the considered area for 10 year

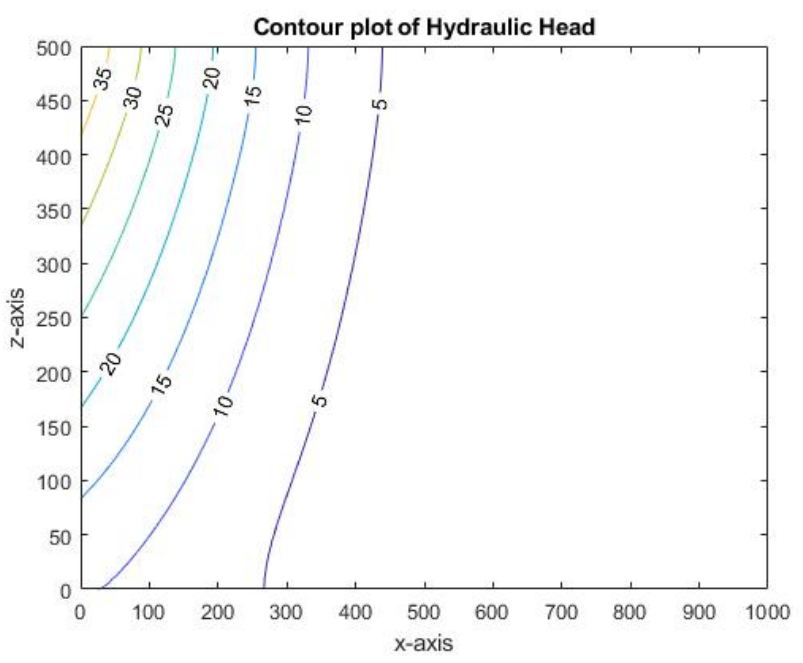

Figure 5. Hydraulic head of the considered area for 10 year

\subsection{Numerical simulation of chloride dispersion}

In the last model, we input the groundwater flow velocity to approximate chloride dispersion. The chemical compound of chloride can be transformed into hypochlorite, then into chlorite, chlorate, and lastly into perchlorate. At the beginning of the time, we assume that the amount of chemical compound in considered area is equal to zero, i.e., $c_{0}=0 \mathrm{~m}$ and boundaries in considered domain suppose that there is no rate of change, then, $g_{L}=g_{T}=g_{R}=g_{B}=0$. The diffusion coefficient in $x$ - and $z$-direction in each models are different due to the mass of compound, so, we assume that the diffusion coefficient $D_{x}=D_{z}=1.5$. The transformed rate are important factor to concentration of pollutant, its valuable between 0 to 1 , then, we set $R=0.001$. The approximate solution is shown in Table 4 and Fig 6-7.

We set the initial and boundary conditions as the same with previous model, considering diffusion coefficient of hypochlorite $D 1_{x}=D 1_{z}=2.0$ and rate of hypochlorite dispersion $R_{1}=0.25$. The approximate solution is shown in Table 5 and 


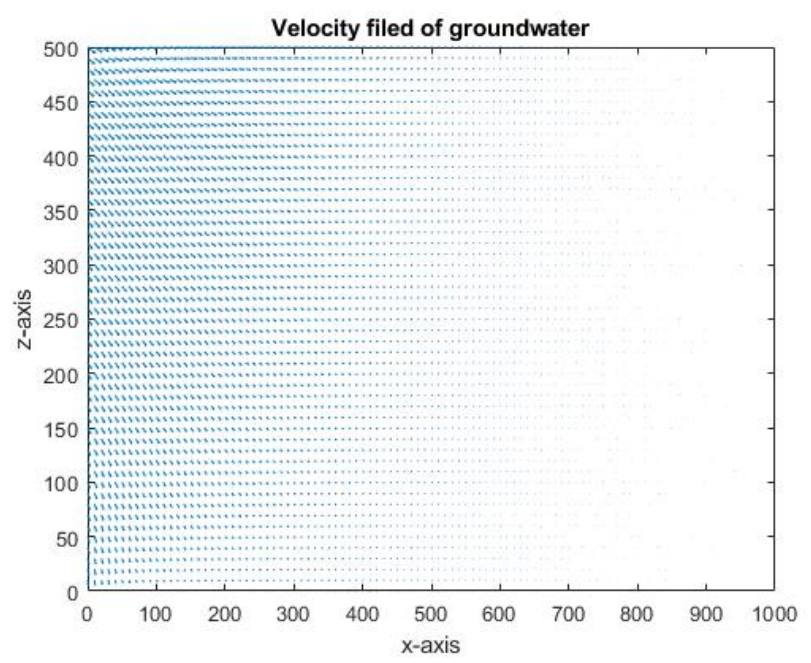

Figure 6. The groundwater flow direction for 10 years

Table 4. The approximated chloride where $z=50 \mathrm{~m}$.

\begin{tabular}{lllllll}
\hline \multicolumn{7}{c}{$c(x, z, t)$} \\
\hline$t$ & $x=0$ & $x=20$ & $x=40$ & $x=60$ & $x=80$ & $x=90$ \\
\hline 3 & 0.0000 & 0.0000 & 0.0001 & 0.0013 & 0.0123 & 0.0305 \\
5 & 0.0000 & 0.0001 & 0.0008 & 0.0060 & 0.0326 & 0.0657 \\
7 & 0.0002 & 0.0005 & 0.0026 & 0.0061 & 0.0285 & 0.0561 \\
10 & 0.0012 & 0.0020 & 0.0070 & 0.0271 & 0.0909 & 0.1516 \\
\hline
\end{tabular}

Fig 8-9.

Table 5. The approximated hypochlorite where $z=50 \mathrm{~m}$.

\begin{tabular}{lllllll}
\hline \multicolumn{7}{c}{$\phi(x, z, t)$} \\
\hline$t$ & $x=0$ & $x=20$ & $x=40$ & $x=60$ & $x=80$ & $x=90$ \\
\hline 3 & 0.0000 & 0.0000 & 0.0001 & 0.0006 & 0.0032 & 0.0064 \\
5 & 0.0001 & 0.0002 & 0.0006 & 0.0025 & 0.0089 & 0.0154 \\
7 & 0.0005 & 0.0008 & 0.0020 & 0.0060 & 0.0172 & 0.0273 \\
10 & 0.0027 & 0.0034 & 0.0093 & 0.0219 & 0.0485 & 0.0687 \\
\hline
\end{tabular}

We set the initial and boundary conditions as the same with previous model, considering diffusion coefficient of chlorite $D 2_{x}=D 2_{z}=0.5$ and rate of chlorite dispersion $R_{2}=0.01$. The approximate solution as shown in Table 6 Fig 10-11.

Table 6. The approximated chlorite where $z=50 \mathrm{~m}$.

\begin{tabular}{lllllll}
\hline \multicolumn{7}{c}{$\eta(x, z, t)$} \\
\hline$t$ & $x=0$ & $x=20$ & $x=40$ & $x=60$ & $x=80$ & $x=90$ \\
\hline 3 & 0.0000 & 0.0000 & 0.0000 & 0.0000 & 0.0001 & 0.0003 \\
5 & 0.0000 & 0.0000 & 0.0000 & 0.0001 & 0.0004 & 0.0006 \\
7 & 0.0000 & 0.0000 & 0.0001 & 0.0002 & 0.0007 & 0.0011 \\
10 & 0.0001 & 0.0001 & 0.0002 & 0.0006 & 0.0013 & 0.0019 \\
\hline
\end{tabular}

We set the initial and boundary conditions as the same with previous model, considering diffusion coefficient of chlorate $D 3_{x}=D 3_{z}=2.5$ and rate of chlorate dispersion $R_{3}=0.4$. The approximate solution as shown in Table 7 and Fig 12-13.

We set the initial and boundary conditions as the same with previous model, considering diffusion coefficient of perchlorate $D 4_{x}=D 4_{z}=1$ and rate of perchlorate dispersion $R_{4}=0.005$. The approximate solution as shown in Table 8 and Fig 14-15.
Table 7. The approximated chlorate where $z=50 \mathrm{~m}$.

\begin{tabular}{lllllll}
\hline \multicolumn{7}{c}{$\sigma(x, z, t)$} \\
\hline$t$ & $x=0$ & $x=20$ & $x=40$ & $x=60$ & $x=80$ & $x=90$ \\
\hline 3 & 0.0000 & 0.0000 & 0.0000 & 0.0000 & 0.0004 & 0.0011 \\
5 & 0.0000 & 0.0000 & 0.0001 & 0.0003 & 0.0017 & 0.0039 \\
7 & 0.0001 & 0.0001 & 0.0004 & 0.0019 & 0.0087 & 0.0171 \\
10 & 0.0004 & 0.0004 & 0.0007 & 0.0025 & 0.0097 & 0.0184 \\
\hline
\end{tabular}

Table 8. The approximated chlorate where $z=50 \mathrm{~m}$.

\begin{tabular}{lllllll}
\hline \multicolumn{7}{c}{$\xi(x, z, t)$} \\
\hline$t$ & $x=0$ & $x=20$ & $x=40$ & $x=60$ & $x=80$ & $x=90$ \\
\hline 3 & 0.0000 & 0.0000 & 0.0000 & 0.0000 & 0.0002 & 0.0003 \\
5 & 0.0000 & 0.0000 & 0.0000 & 0.0001 & 0.0003 & 0.0004 \\
7 & 0.0000 & 0.0000 & 0.0001 & 0.0002 & 0.0005 & 0.0007 \\
10 & 0.0001 & 0.0002 & 0.0003 & 0.0005 & 0.0009 & 0.0013 \\
\hline
\end{tabular}

\section{Discussion}

In our simulation, we assume that the hydraulic head drive the groundwater flow from the higher hydraulic head level zone to the lower zone, the result of simulation for 10 years have been shown in Table 1 and Figure 3-4. The figures shown that the hydraulic head at the surface area is higher than the deep area. The hydraulic head is transformed to be the groundwater flow direction as shown in Fig 5. The direction has shown that groundwater flow from high to lower hydraulic head. The result has been plug into the five chloride compound dispersion models. We can measure the total chloride, hypochloride, chlorite, chlorate and perchlorate pollutant levels at 10 years as shown Table 4-8 and Fig 6-16. The figure shows that the amount of groundwater changes directly,over time the substance has been less than reactant. The approximated chloride compound is compared in Fig 16-20. In Fig 16-20, we can see that the simulation for 2 to 4 years tell us the trend of graph are the same and a little bit increasing, after that, for 4 to 6 years, they are slightly increase. Finally, in 6 to 10 years we obtained that the graph is stable.

\section{Conclusion}

A Mathematical model of horizontal average groundwater pollutant measurement with several substances due to chemical reaction is introduced. The first model is the two-dimensional transient groundwater flow model which provides the hydraulic head. The second is the groundwater flow velocity model which provides the groundwater flow in $x$ - and $z$-directions. The third model is the two-dimensional horizontal averaged contaminated chloride dispersion model which provides the concentration of chloride and their substances. A method to set up the initial and boundary conditions of transient groundwater flow model is proposed. The computed hydraulic head is transformed to be the groundwater flow velocity by using the second model. The results in second model will be input into the last model as a field data. The concentration of chloride and their substances are obtained by the third model. The hydraulic head of the first model is approximated by an explicit finite difference method. An explicit finite difference technique is used to obtain the groundwater flow velocity of the 
second model. A forward time-centered space finite difference technique is used to approximate the concentration of chloride and their substances. The groundwater quality is affected by the chloride release by the landfill. The proposed simulations show that the different levels of hydraulic head have a small effect on the overall groundwater quality level. In our simulation.It is found that the main groundwater quality factor are pollutant concentration level around the landfill and transform rate.

\section{Acknowledgment}

This paper is supported by the Centre of Excellence in Mathematics, the Commission on Higher Education, Thailand. The authors greatly appreciate valuable comments received from the anonymous reviewers.

\section{References}

[1] Linsay McCallum, Stefanie Lip, Sandosh Padmanabhan, The hidden hand of chloride in hypertension. Pflugers Arch - Eur J Physiol, 467 (2015), 595 - 603.

[2] Majumdar D. (2003). The Blue Baby Syndrome Nitrate Poisoning in Humans, Resonance, 8(10), 20-30.

[3] Yamashita N., Sugio S. (2000), Numerical Simulation of Nitrate Transport with Unsaturated Flow Condition in Volcanic Soils. In: Sato K., Iwasa Y. (eds) Groundwater Updates. Springer, Tokyo.

[4] Gardenas, A I , Hopmans, J W , Hanson, B R , and Simunek J. (2005). Two-dimensional modeling of nitrate leaching for various fertigation scenarios under microirrigation.

[5] Dehghan M., (2004). Weighted finite difference techniques for the one-dimensional advection-diffusion equation. Applied Mathematics and Computation, 147(2), 307-319.

[6] Suebyat K and Pochai N, (2018), Numerical Simulation for a Three-Dimensional Air Pollution Measurement Model in a Heavy Traffic Area under the Bangkok SkyTrain Platform. Abstract and Applied Analysis.

[7] Kraychang W and Pochai N, (2016), Implicit Finite Difference Simulation of Water Pollution Control in a Connected Reservoir System. International Journal of Applied Mathematics, 46 (1).

[8] K. Halil and M. Tammer Ayvaz, Transient groundwater modeling using spreadsheets. Advances in Engineering Software, 36 (2005) 374-384.

[9] N. Pochai and N. Pongnu, Numerical solution of groundwater measurement using alternating direction methods.
Journal of Interdisciplinary Mathematics, vol 20 (2017), No. 2, 513-541.

[10] N. Pochai, Numerical Treatment of a Modified MacCormack Scheme in a Nondimensional Form of the Water Quality Models in a Nonuniform Flow Stream. Journal of Applied Mathematics, Volume 2014, Article ID 274263, 8 pages .

[11] N. Pochai, S. Tangmanee, L. J. Crane, and J. J. Miller, A mathematical model of water pollution control using the finite element method. Proceedings in Applied Mathematics and Mechanics, vol 6 (2006), no. 1, 755-756.

[12] N. Pochai, S. Tangmanee, L. J. Crane, and J. J. Miller, A water quality computation in the uniform channel. Journal of Interdisciplinary Mathematics, vol 11 (2008), no. 6, 803-814.

[13] N. Pochai and C. Sornsri, A Non-dimensional Form of Hydrodynamic Model with Variable Coefficients in a Uniform Reservoir Using Lax-Wendroff Method. Procedia Engineering, 8 (2011), 89-93.

[14] G. Amin, Explicit and implicit forms of differential quadrature method for advection-diffusion equation with variable coefficients in semi-infinite domain. Journal of Hydrology, 541 (2016) 935-940.

[15] N. Pochai and R. Deepana, A Numerical Computation of Water Quality Measurement in a Uniform Channel Using a Finite Difference Method. Procedia Engineering, 8 (2011) 85-88.

[16] Cryer CW, On the approximate solution of free boundary problems using finite difference. Journal of the Association for Computing Machinary, vol 17 (1970), 397-411.

[17] Bardet JP and Tobita T, A practical method for solving free-surface seepage problems. Computers and Geotechnics, 29 (2002), 451-475.

[18] Ayvaz MT, Tuncan M, Karahan H and Tuncan A, An extended presure application for transient seepage problems with a free surface. Journal of Porous Media, 8 (2005), $613-625$.

[19] Desai CS and Li GC, A residual flow procedure and application for free surface flow in porous media. Advances in Water Resources, 6 (1983), 27-35

[20] Kikuchi N, An analysis of the variational inequalities of seepage flow by finite element methods. Quarterly of Applied Mathematics, 35 (1977), 149-163.

[21] Tatfur G, Swiatek D, Wita A and Singh VP, Case study: Finite element method and artificial neural network models for flow through Jeziorsko earthfill dam in Poland. Journal of Hydraulic Engineering, 191 (2005), 431-440. 


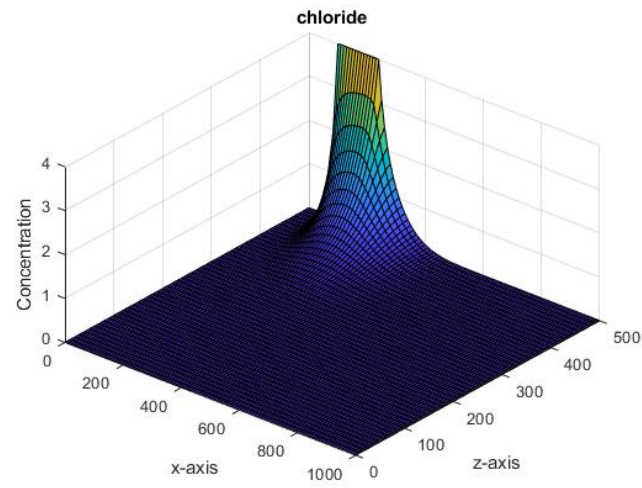

Figure 7. Surface plot of chloride concentration for 10 years

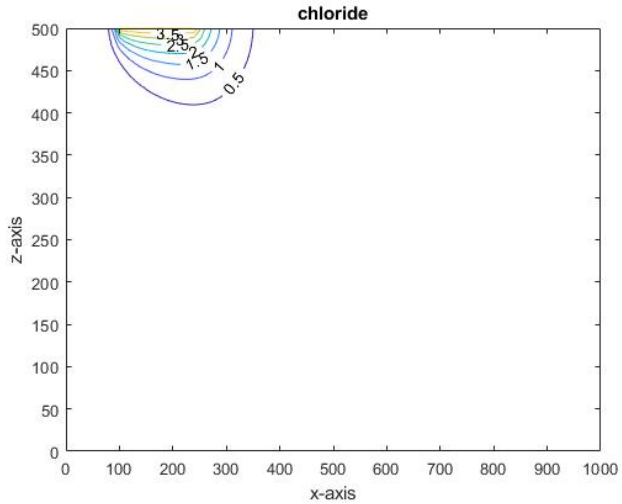

Figure 8. Contour plot of chloride concentration for 10 years

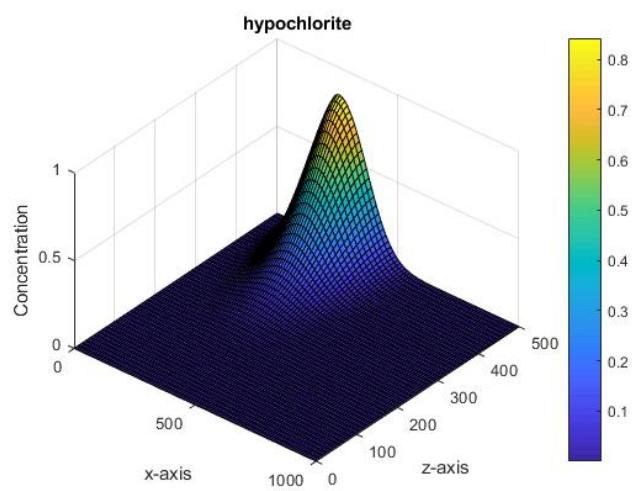

Figure 9. Surface plot of hypochlorite concentration for 10 years

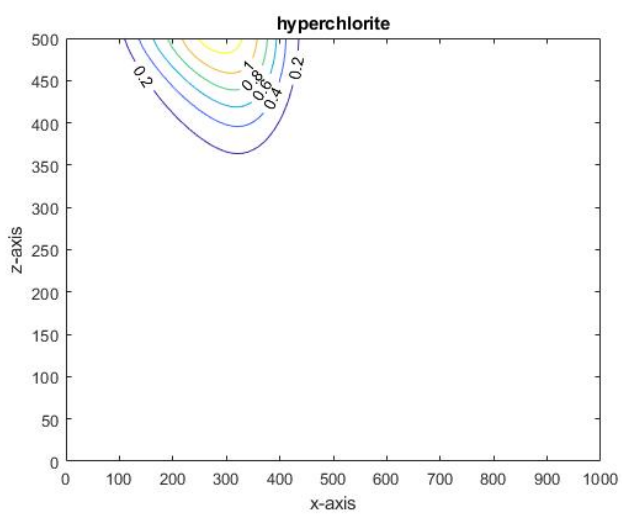

Figure 10. Contour plot of hypochlorite concentration for 10 years

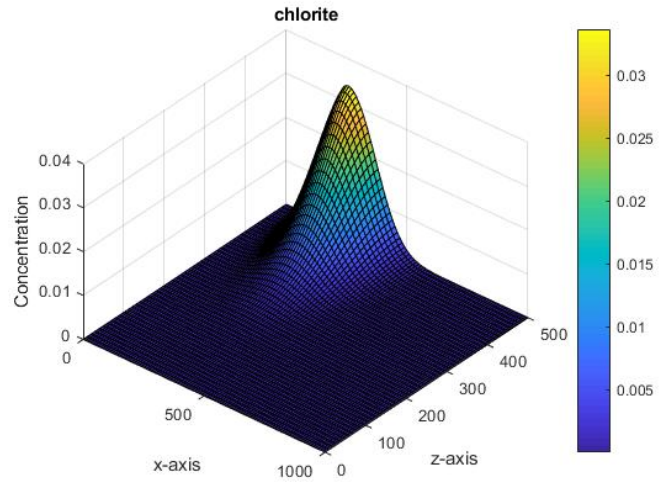

Figure 11. Surface plot of chlorite concentration for 10 years

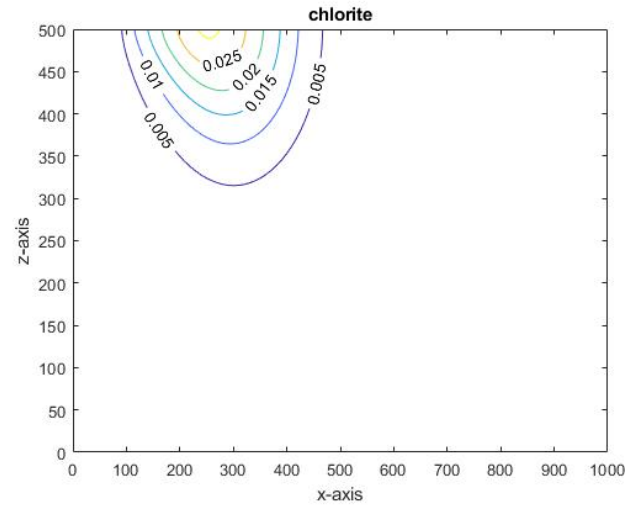

Figure 12. Contour plot of chlorite concentration for 10 years

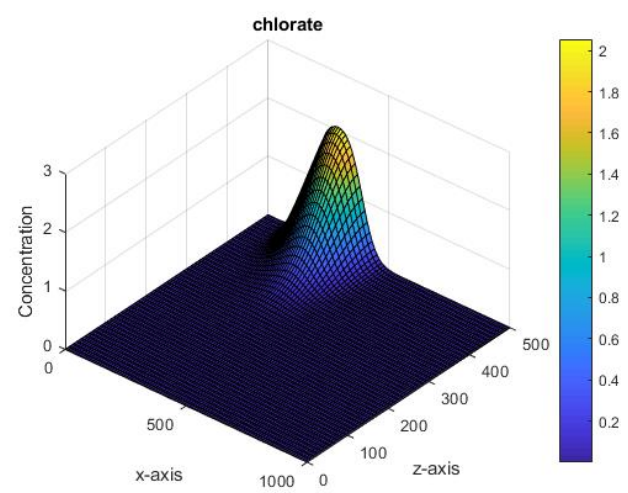

Figure 13. Surface plot of chlorate concentration for 10 years

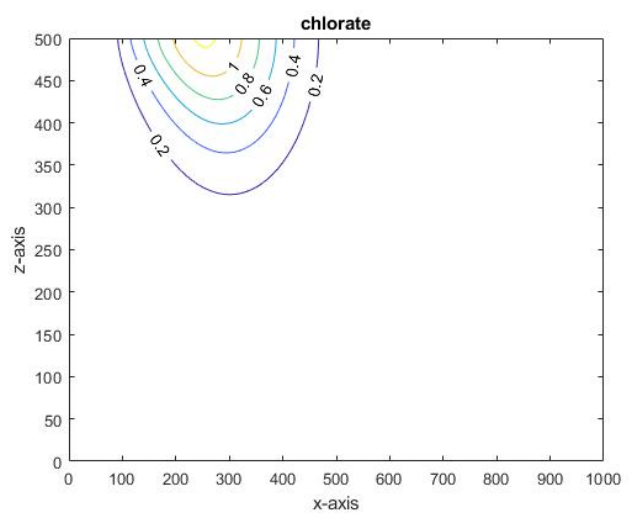

Figure 14. Contour plot of chlorate concentration for 10 years 


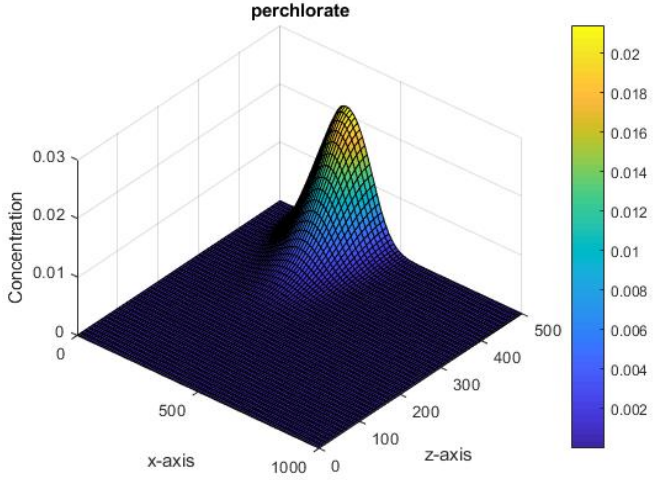

Figure 15. Surface plot of perchlorate concentration for 10 years

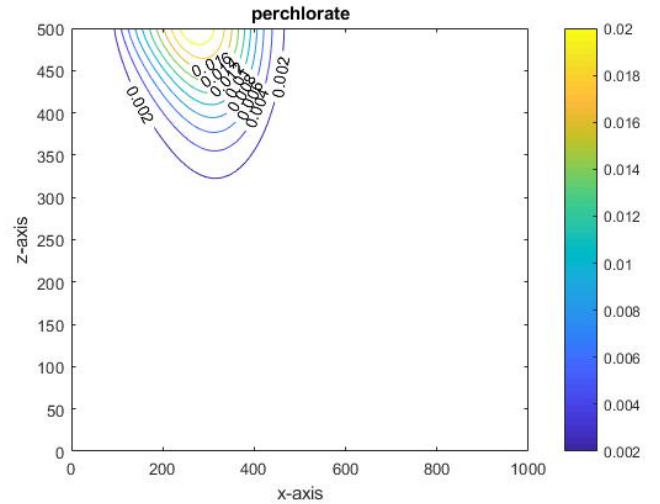

Figure 16. Contour plot of perchlorate concentration for 10 years

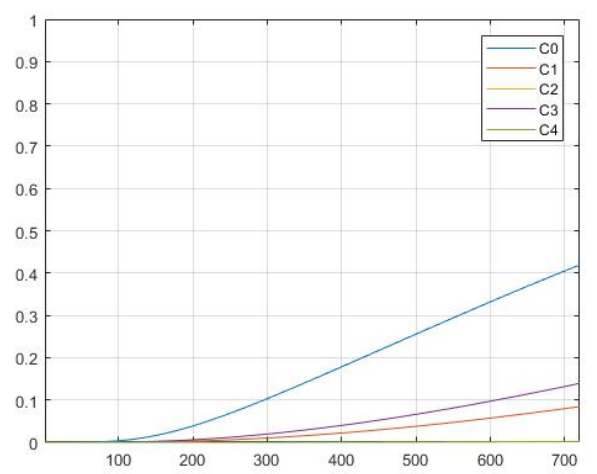

Figure 17. Comparison of chloride compound levels along 2 years

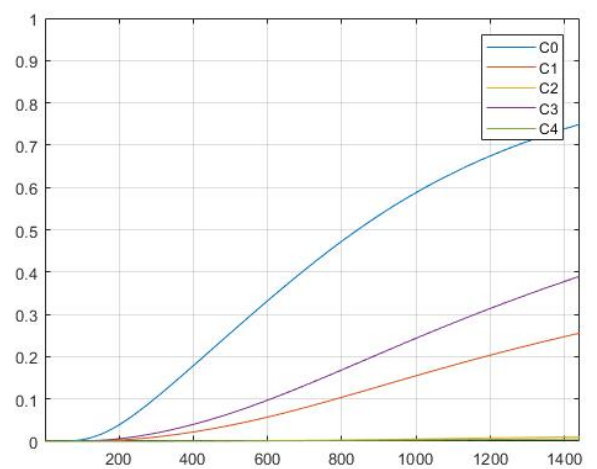

Figure 18. Comparison of chloride compound levels along 4 years

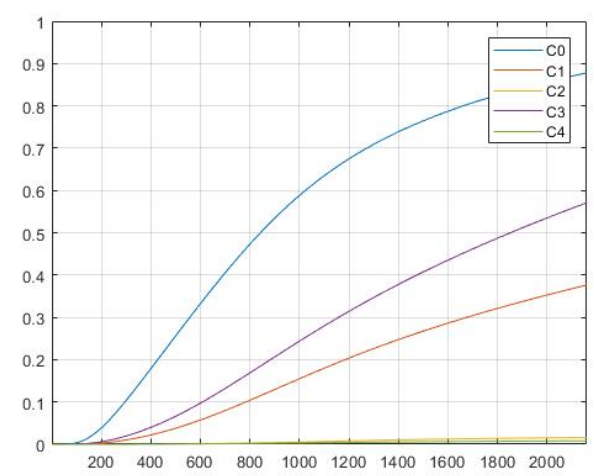

Figure 19. Comparison of chloride compound levels along 6 years

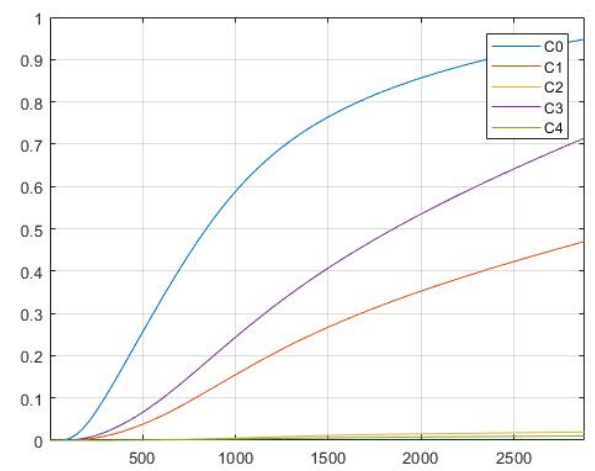

Figure 20. Comparison of chloride compound levels along 8 years

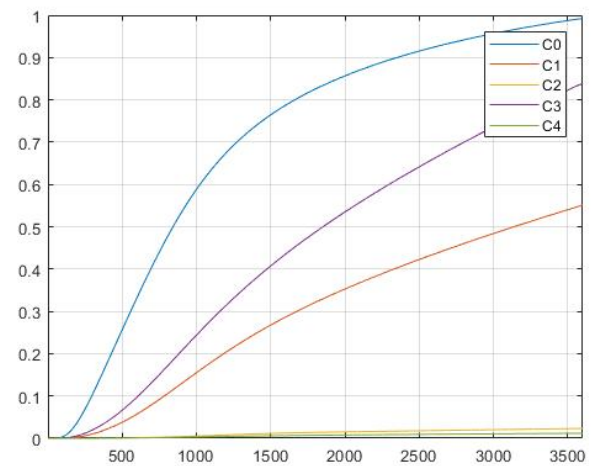

Figure 21. Comparison of chloride compound levels along 10 years with $\mathrm{C} 0=$ total chloride, $\mathrm{C} 1$ = hypochloride, $\mathrm{C} 2=$ chlorite, $\mathrm{C} 3=$ chlorate, $\mathrm{C} 4=$ perchlorate. 